\title{
Clinical application of concentrate growth factors combined with bone substitute in Alveolar ridge preservation of anterior teeth
}

\section{Dilinuer Keranmu}

the First Affiliated Hospital of Xinjiang Medical University (Affiliated Stomatological Hospital). Research Institute of Stomatology of Xinjiang Uygur Autonomous Region

\section{Nijiati Nuermuhanmode}

the First Affiliated Hospital of Xinjiang Medical University (Affiliated Stomatological Hospital). Research Institute of Stomatology of Xinjiang Uygur Autonomous Region

\section{Ailimaierdan Ainiwaer}

the First Affiliated Hospital of Xinjiang Medical University (Affiliated Stomatological Hospital). Research Institute of Stomatology of Xinjiang Uygur Autonomous Region

\section{Adili Moming}

Stomatological Institute of Fujian Guoyao Oriental Hospital

\section{Gu Li}

the First Affiliated Hospital of Xinjiang Medical University (Affiliated Stomatological Hospital). Research Institute of Stomatology of Xinjiang Uygur Autonomous Region

\section{Dilidaer Taxifulati}

the First Affiliated Hospital of Xinjiang Medical University (Affiliated Stomatological Hospital). Research Institute of Stomatology of Xinjiang Uygur Autonomous Region

\section{Wang Shan}

the First Affiliated Hospital of Xinjiang Medical University (Affiliated Stomatological Hospital). Research Institute of Stomatology of Xinjiang Uygur Autonomous Region

\section{Wang Ling ( $\square$ crystalWL272@126.com)}

the First Affiliated Hospital of Xinjiang Medical University (Affiliated Stomatological Hospital). Research Institute of Stomatology of Xinjiang Uygur Autonomous Region

\section{Research Article}

Keywords: Alveolar bone, Alveolar ridge preservation(ARP), concentrate growth factors ( CGF), Bio-oss bone powder

Posted Date: December 27th, 2021

DOI: https://doi.org/10.21203/rs.3.rs-1174819/v1 
License: (c) (i) This work is licensed under a Creative Commons Attribution 4.0 International License. Read Full License

Version of Record: A version of this preprint was published at BMC Oral Health on March 3rd, 2022. See the published version at https://doi.org/10.1186/s12903-022-02091-8. 


\section{Abstract}

Objective: To investigate the clinical effect of concentrated growth factors (CGF) combined with Bio-oss bone powder on Alveolar ridge preservation (ARP) during implantology. Methods: A total of 38 patients were selected and randomly divided into 2 groups, with 19 cases in each group. The extraction sockets were filled with Bio-oss bone powder with or without CGF. VAS pain score was recorded within 1 week and Landry wound healing index (LWHI) was recorded at 1, 2 and 3 weeks after operation. CBCT was taken 3 and 6 months after operation to measure and compare the changes of vertical height, width and gray value of alveolar bone at extraction site. The changes of alveolar bone contour were observed clinically and compared between the two groups. Results: The VAS score of CGF group was lower than control group on the 1 st and 3rd day after operation $(P<0.05)$. The LWHI of CGF group was higher than control group 1 week after operation $(P<0.05)$. The absorption of the labial and palatal plates height and the width in the CGF group was significantly less than the control group at 3 months $(P<0.05)$. The gray value of alveolar bone in CGF group was significantly higher than control group at 3 months $(P<0.05)$. There was no significant difference in new bone contour between the two groups $(P>0.05)$. 94.7\% cases in CGF group did not undergo bone grafting, which was significantly higher than control group (78.9\%). Conclusions: The use of CGF combined with Bio-oss bone powder can help to reduce postoperative pain at the early stage of healing, form sufficient keratinized gingival tissue, effectively maintain the height and width of alveolar bone in the three-dimensional direction and provide good conditions for implant repair in the future.

\section{Background}

Implant restoration has been gradually accepted by more and more patients, because there is no damage to the adjacent teeth. Besides, implants have the same function as natural teeth which can bear and transmit masticatory force well. However, the absorption of alveolar bone after tooth extraction limits the application of implant. Alveolar bone can be absorbed to different degrees after tooth extraction, especially after 3-6 months. The alveolar ridge could lose $29 \%-63 \%(2.46-4.56 \mathrm{~mm})$ of its original width and $11 \%-22 \%(0.8-1.5 \mathrm{~mm})$ of its original height, ${ }^{1}$ which will lead to the deficiency of alveolar ridge bone and affect the long-term use and aesthetic effect of implants. Therefore, how to preserve the mass of alveolar bone is the critical problem of implant repair. ${ }^{2}$

The concept of ARP was first proposed in 2005. It refers to the protective intervention on the sites that need delayed implant restoration while extracting the tooth. ${ }^{3}$ The morphology of soft and hard tissue can be preserved to the greatest extent by reducing the bone loss of extraction socket and accelerating bone regeneration. There are many different ARP techniques and various types of materials, such as autogenous bone, ${ }^{4}$ allografts, ${ }^{5}$ xenografts and platelet concentrates ${ }^{6-7}$. The main goal of the bone graft material is to serve as a scaffold and maintain a space for bone ingrowth, blood vessels formation, to support soft tissues and to improve the quality and quantity of regenerated bone. ${ }^{8}$ Deproteinized bovine bone graft (Bio-Oss) is a classical xenogeneic bone graft material, which is made from deorganized 
bovine limb bone, and generally biocompatible and structurally similar to human bone. Bio-oss bone powder has been developed as the preferred alternative bone material and used in restoration of bone defects around implants, ridge preservation after extraction, ${ }^{9}$ maxillary sinus augmentation, ${ }^{10}$ treatment of cyst and generally resulted in new attachment and cementum formation when compared to ungrafted sites.

In recent years, concentrated platelets have been used in wound healing because of their high growth factor content. Among the preparations, CGF, platelet-rich plasma (PRP), platelet-derived growth factor, transforming growth factor beta and platelet-rich fibrin (PRF) currently used for the regeneration and reconstruction of bone and connective tissues are. CGF is a new generation of plasma extract prepared from patients' own venous blood by special centrifugation. CGF was first proposed by Sacco in 2006, which contains high concentrations of a variety of growth factors and fibrin. ${ }^{11}$ The preparation process is simple, without the risk of cross infection and allergic reaction, it is safe and reliable for clinical use. CGF has been widely used in the fields of oral implantation, maxillary sinus lifting, treatment of jaw cysts and promotion of fracture healing. ${ }^{12-15}$

However, the researchs on the combined application of CGF and Bio-oss bone powder in ARP were limited. Therefore, the aim of this study was to perform a clinical and radiographic evaluation of the ARP technique using CGF and Bio-oss bone powder, to observe the conditions of the alveolar ridge bone after tooth extraction and evaluate the application value of CGF combined with Bio-oss bone powder in alveolar bone increment.

\section{Materials And Methods}

\section{Patient population and enrollment}

38 patients (38 teeth in total) who underwent single root extraction at the Outpatient department of Stomatology from October 2020 to May 2021 were collected. 38 extraction sites were randomly divided into CGF group and control group, 19 cases in each. All patients were informed about the potential benefits and risks of surgery, as well as alternative treatment options, and volunteered to participate in and signed an informed consent. The protocol of this study was consistent with the ethical guidelines of the Declaration of Helsinki and was approved by the Ethics Committee of the First Affiliated Hospital of Xinjiang Medical University (Ethics approval No.: 210723-08). All patients voluntarily participated in the study and signed informed consent.

Inclusion criteria were as follows (1) Patients aged 18 and over; (2) Teeth cannot be retained due to severe caries, chronic periapical periodontitis or trauma, and need implant restoration; (2) All extraction sites had adjacent teeth with good periodontal condition; (3) At least 2 or more bone plates exists at the extraction site.; (4) Without serious systemic diseases, psychosis and epilepsy; (5) Non smoking and good compliance. 
Exclusion criteria were as follows (1) Patients with acute periodontal or periapical infection; (2) Buccal and lingual alveolar bone resorption exceeds $25 \%$ of root length; (3) With severe hypertension, diabetes, kidney and liver diseases; (4) Pregnant patients; (5) Patients with a history of radiotherapy and chemotherapy.

\section{Preoperative work-up}

Preoperative examination including general conditions, routine blood tests, oral hygiene, occlusal relationship, etc. CBCT was taken to measure the height and width of alveolar bone. (Figure 1). We identified the most coronal points of the extraction plate on the buccal side and the palatal/lingual side as $b$ and $p$, respectively, while the most coronal extensions of the healed ridge in these two sections were b0 and p0, respectively. Vertical resorption included both the buccal side $(\mathrm{Hb})$ and the palatal/lingual side ( $\mathrm{Hp})$. $\mathrm{Hp}$ and $\mathrm{Hb}$ were divided into W1, W2 and W3, and the horizontal ridge width was measured and recorded.

Preparation of CGF: Venous blood was collected from each patients in CGF group using sterile vacuum tubes without additive. Then the tubes with whole blood ( $4 \mathrm{~mL}$ in each) were immediately centrifuged by Medifuge(Silfradent, Italy) at fixed temperature. After centrifugation, CGF gel represented as the buffy coat in the middle layer and was carefully isolated from the red blood cell clots.(Figure 2) One of the prepared CGF was pressurized to remove the liquid components to make CGF membrane for later use.

\section{Surgical procedure}

Mouth rinsing was performed 3 times with $0.2 \%$ chlorhexidine solution before operation. Under local infiltration anesthesia with articaine, the gingival were separated and the affected tooth was extracted atraumatically. After removing the affected tooth, the periapical lesion was curetted by using bone curettes when the root tip was inflamed and the surgical area was then rinsed with physiological saline. In the CGF group, CGF was cut into small particles, fully mixed with Bio-oss bone powder, and filled into the extraction socket in layers to make it about $2 \mathrm{~mm}$ higher than the crest of the surrounding alveolar ridge, the wound was completely covered with CGF membrane and then Collagen membrane (HAIAO). Finally, the flap was repositioned coronally and sutured tightly with non-resorbable sutures. In the control group, Bio-oss bone powder was filled into the extraction socket to make it about $2 \mathrm{~mm}$ higher than the crest of the surrounding alveolar ridge, the wound was completely covered with Collagen membrane and the flap was sutured.(Figure 3)

\section{Postoperative examination and treatment}

After the grafting surgery, systemic antibiotics were prescribed to all patients 1 day before operation for 5 days. Visual analogue scale (VAS) was used to measure the degree of postoperative pain on day 1,3 , and 7. The sutures were removed after 1 week. According to the color of the gingiva, the presence or absence of granulation tissue, bleeding, pyorrhea and epithelial formation, the Landry Wound Healing Index (LWHI) was recorded at 1, 2 and 3 weeks. ${ }^{16}$ CBCT was taken after 3 and 6 months to compare the changes of alveolar bone height, width and gray value of socket between the two groups. The contour of 
alveolar bone was recorded and compared with the adjacent teeth and the same teeth on the other side after 3 and 6 months.

\section{Statistical analysis}

Measurements were recorded in a spreadsheet in Excel 2013 (Microsoft Corporation, WA) and were then analyzed using SPSS 20.0 (SPSS Inc., IL). Data are given as mean \pm SD and were evaluated via a Shapiro-Wilk test to assess distribution normality. Normally distributed data were compared via t-test or analysis of variance (ANOVA), while non-normally distributed data were compared via Mann- Whitney U test. A significance level of $a=0.05$ was used for all analyses.

\section{Results}

\section{General observation}

In total, we identified 38 patients meeting study enrollment criteria, 19 cases in each group, 23 females and 15 males, with a mean age of $(28.89 \pm 2.7)$ years, all of them completed all trial protocols and subsequent implant restoration. All sockets healed uneventfully, and no signs of acute infection or excessive inflammatory response were observed during the 6-month clinical healing period. The contour of alveolar bone in the trial group was more favorable for the prosthesis esthetics. All implants did not have peri-implantitis or loose or lost implant. There was no significant differences in average patient age between the two groups.

\section{Postoperative pain and soft tissue healing}

The VAS score of CGF group was lower than control group on the 1 st day after operation $(P<0.05)($ Table 1). The LWHI of the CGF group and the control group at 1, 2 and 3 weeks after operation is shown in Table 2. The LWHI of CGF group was higher than control group 1 week after operation $(P<0.05)$.

Table 1

VAS scores of the two groups

\begin{tabular}{|llll|}
\hline Group & \multicolumn{3}{l|}{ Visual analogue scale(VAS) } \\
\cline { 2 - 4 } & $\mathbf{1}$ day & $\mathbf{3}$ days & $\mathbf{7}$ days \\
\hline Control Group & $5.61 \pm 0.85$ & $3.03 \pm 0.61$ & $1.17 \pm 0.99$ \\
\hline CGF Group & $4.33 \pm 1.07$ & $3.00 \pm 0.62$ & $1.17 \pm 0.99$ \\
\hline$P$ value & $<0.05$ & 0.893 & 1 \\
\hline
\end{tabular}


Table 2

LWHI of the two groups

\begin{tabular}{|llll|}
\hline \multicolumn{4}{|l|}{ Landry Wound Healing Index $(\mathrm{LWHI})$} \\
\hline Group & 1 week & 2 weeks & 3 weeks \\
\hline Control Group & $2.50 \pm 0.62$ & $3.88 \pm 0.58$ & $4.83 \pm 0.38$ \\
\hline CGF Group & $3.94 \pm 0.64$ & $4.83 \pm 0.38$ & $5.00 \pm 0.00$ \\
\hline$P$ value & $<0.05$ & 0.092 & 0.074 \\
\hline
\end{tabular}

\section{CBCT analysis}

The buccal and palatal/lingual absorption of the two groups at 3 and 6 months after tooth extraction is shown in Table 3. The absorption of the buccal and palatal/lingual plates height and the and changes in the two groups ridge width in the two groups at 3 and 6 months after tooth extraction is shown in Table 3 . There were significant differences in buccal and palatal/lingual vertical bone resorption and the ridge width between the two groups at 3 and 6 months after operation $(P<0.05)$. The gray value of alveolar bone in CGF group was significantly higher than control group at 3 months $(P<0.05)$.(Figure 3$)$ (Table 3$)$ 
Table 3

The changes of ridge height,width and grey value between preoperative, 3 and 6 months later in two groups

\begin{tabular}{|c|c|c|c|c|}
\hline \multirow[t]{2}{*}{ Items } & & \multicolumn{2}{|l|}{ Group } & \multirow[t]{2}{*}{$P$ value } \\
\hline & & Control Group & CGF Group & \\
\hline \multirow[t]{3}{*}{$\mathrm{Hb}(\mathrm{mm})$} & preoperative & $17.22 \pm 1.13$ & $17.16 \pm 1.25$ & 0.868 \\
\hline & $3 M$ & $15.66 \pm 1.34$ & $16.86 \pm 1.25$ & $<0.05$ \\
\hline & $6 \mathrm{M}$ & $14.89 \pm 1.53$ & $16.52 \pm 1.26$ & $<0.05$ \\
\hline \multirow[t]{3}{*}{$\mathrm{Hp}(\mathrm{mm})$} & preoperative & $16.85 \pm 1.34$ & $16.68 \pm 1.36$ & 0.713 \\
\hline & $3 M$ & $14.01 \pm 1.36$ & $16.61 \pm 1.37$ & $<0.05$ \\
\hline & $6 \mathrm{M}$ & $13.20 \pm 1.25$ & $15.18 \pm 1.37$ & $<0.05$ \\
\hline \multirow[t]{3}{*}{$\mathrm{W} 1(\mathrm{~mm})$} & preoperative & $6.37 \pm 0.76$ & $6.41 \pm 0.60$ & 0.866 \\
\hline & $3 \mathrm{M}$ & $4.43 \pm 0.67$ & $5.91 \pm 0.67$ & $<0.05$ \\
\hline & $6 \mathrm{M}$ & $3.22 \pm 0.69$ & $5.10 \pm 0.71$ & $<0.05$ \\
\hline \multirow[t]{3}{*}{ W2(mm) } & preoperative & $8.10 \pm 0.60$ & $8.26 \pm 0.55$ & 0.432 \\
\hline & $3 \mathrm{M}$ & $5.49 \pm 1.05$ & $7.60 \pm 0.74$ & $<0.05$ \\
\hline & $6 \mathrm{M}$ & $4.82 \pm 0.99$ & $6.93 \pm 0.71$ & $<0.05$ \\
\hline \multirow[t]{3}{*}{ W3(mm) } & preoperative & $10.76 \pm 0.60$ & $10.94 \pm 0.51$ & 0.32 \\
\hline & $3 \mathrm{M}$ & $9.10 \pm 0.86$ & $10.20 \pm 0.59$ & $<0.05$ \\
\hline & $6 \mathrm{M}$ & $8.51 \pm 0.91$ & $9.46 \pm 0.43$ & 0.28 \\
\hline \multirow[t]{3}{*}{ grey value(Hu) } & preoperative & $1363.72 \pm 38.21$ & $1360.22 \pm 53.04$ & 0.822 \\
\hline & $3 \mathrm{M}$ & $2055.67 \pm 120.82$ & $2270.89 \pm 42.29$ & $<0.05$ \\
\hline & $6 \mathrm{M}$ & $2182.00 \pm 109.78$ & $2289.11 \pm 39.01$ & 0.29 \\
\hline
\end{tabular}

\section{New bone contour evaluation}

Clinical observation showed that the attached gingival of the extraction site in the CGF group was sufficient, with normal color and plump appearance, and no infection or necrosis was observed. All the implants were successfully completed. In the control group, the extraction site healed well, but the alveolar crest showed different degrees of atrophy, and the attached gingival was relatively narrow. There was no significant difference in new bone contour between the two groups. 


\section{Bone grafting at the extraction site}

After 6 months of follow-up, while only 1 case in the CGF group underwent bone increment by GBR, 3 cases in the control group underwent bone increment and 1 case received connective tissue transplantation. $94.7 \%$ cases in CGF group did not undergo bone grafting, which was significantly higher than control group (78.9\%).

\section{Discussion}

The alveolar ridge bone resorption and soft tissue shrinkage are the main problems after tooth extraction, which compromise the alveolar ridge esthetics and function. To achieve successful implantation and long-term results, sufficient bone volume, keratinized gingiva around the implant neck and proper occlusal relationship should be achieved. Therefore, it is very important to preserve as much alveolar bone as possible at the time of tooth removal to reduce edentulous ridge resorption rate and bone remodelling after tooth extraction. ${ }^{17}$ Compared with the natural healing, ARP has significant advantages in preserving the size and contour of alveolar bone, especially in the maxilla. ${ }^{18}$

Most studies on the dimensional changes of soft and hard tissues after tooth extraction suggested that the ideal bone graft material should not only have osteoconductive properties but also promote osteoinduction and osteogenesis. ${ }^{19}$ Only autologous bone has these three characteristics and is still considered to be the gold standard for bone augmentation surgery. ${ }^{20}$ However, due to the additional site and prolonged time of operation, the morbidity of donor side, limited autogenous bone availability and postoperative discomfort, bone substitutes are more commonly used for bone regeneration. Bone graft materials are chosen based on their ability to serve as a scaffold, maintain space for new bone ingrowth and possess osteoconductive activity. ${ }^{21}$

Bio-oss bone powder has strong hydrophilicity, high biocompatibility and good plasticity, it can promote the adhesion of osteoblasts to bone, plays important role as a scaffold, and has a very low risk of causing host allergy, inflammation and immune reactions. Bio-oss bone powder can guide and promote the formation of new bone. Histological studies have found that the rate of new bone formation is about $26.0 \% \pm 23.7 \%$ when Bio-oss bone powder was used alone, and it can even reach $48.3 \%{ }^{22-23}$ Although Bio-Oss bone powder has been proven to hane reliable osteogenesis effect as a bone substitute material, ${ }^{24}$ Bio-oss bone powder lacks osteogenesis and osteoinduction. The part where Bio-oss bone powder directly contacts the bone surface will first lead to the formation of new bone, while it takes one

year at the peripheral part without direct contact. ${ }^{25}$ The use of Bio-oss bone powder alone will delay bone healing and prolong osteogenesis time to a certain extent. Therefore, it is significant to explore a material to promote the osteoinduction of bone substitute materials.

CGF is a new generation of platelet concentrate after PRP and PRF. The production of CGF requires variable speeds to separate blood cells from fibrin-rich blocks, which are denser and contain a higher 
concentration of growth factors than PRF. ${ }^{15}$ This results in a better regenerative capacity and greater versatility. CGF contains a large amount of platelet-derived growth factor (PDGF), vascular endothelial growth factor (VEGF), epidermal growth factor (EGF), insulin-like growth factor (IGF), fibroblast growth factor (FGF), bone morphogenetic proteins (BMPs) and metastatic growth factors- $\beta$ ( TGF- $\beta$ ) etc. Among which, FGF can accumulate macrophages, fibroblasts and other cells to the wound site through chemotaxis, thereby promoting wound healing. ${ }^{26-27}$ BMP can mediate osteogenesis alone and promote the formation of bone matrix and form calcified bone tissue when mix with other bone growth factors. TGF- $\beta$, as an important regulatory factor in the process of bone formation and remodeling, controls inflammation through synthetic fibrous connective tissue and local vascular proliferation, and also induces regeneration of alveolar bone. ${ }^{28}$

In this study, none of the patients experienced rejection or wound infections around the grafting region, which indicated that the deproteinized bovine bone and CGF were safe and biocompatible. Due to the dispersive nature and small particles of Bio-oss bone powder, leakage often occurs, so it is necessary to cover the wound with a collagen membrane, and the gingival flap is tightly sutured to prevent the leakage of bone powder. In this study, CGF was cut into small pieces and fully mixed with Bio-Oss bone powder, which was easy to mold. In the meantime, because of the elasticity and adhesion of CGF membrane, leakage rarely occurs even if the tooth extraction wound cannot be closed tightly.

The vertical bone loss of labial buccal bone wall was more obvious than the lingual bone wall due to its thin thickness after tooth extraction. ${ }^{29}$ Similar conditions were observed in our study, so the alveolar crest height was divided into labial and palatal plate height, which were measured and compared respectively. Different from the changes in the control group, the height of labial and palatal plate in CGF group did not change significantly 3 months after operation. Although the width of alveolar ridge decreased, the change was much less than control group. During the implantation operation, some bone powder particles at the tooth extraction in CGF group were seen surrounded by new bone. Furthermore, CBCT showed obvious bone trabecular formation in the operation area. It can be concluded that the use of CGF combined with Bio-oss bone powder for ARP can effectively maintain the volume of the alveolar bone, significantly promote the regeneration of the alveolar tissue, and reduce bone resorption effectively. Therefore, when ready to insert dental implant, the CGF group has better alveolar ridge condition, more bone volume, and better surgical environment. Many other studies have shown that CGF can accelerate bone healing. ${ }^{13,15,30,31,32}$ Among them, Kim et al. ${ }^{13}$ applied CGF in sinus augmentation without any graft materials and confirmed respectively that CGF was effective in promoting healing of bone and can induce new bone formation rapidly. Our previous research showed that the application of CGF in recipient site with a small area of chronic periapical lesions can accelerate the regeneration of alveolar bone and the healing of inflammation, greatly shorten the healing period. ${ }^{30}$ Fang et al. ${ }^{31}$ found that the combinational use of CGFs with Bio-Oss bone powder could promote new bone regeneration without adding exogenous stem cells in bilateral maxillary sinus floor augmentation, which yields effects similar to combining BMSCs with Bio-Oss. Furthermore, Durmuşlar et al. ${ }^{32}$ found that the combined use of CGF and bone graft enhanced the expression of osteogenic related genes and stem cell marker STRO-1, and 
promoted bone regeneration of large defects around implants (about $2.37 \mathrm{~mm}$ in diameter). Together, these results recommend the use of CGF as a restoration material in bony defects.

Sufficient alveolar ridge dimensions is important for implant placement, it is also necessary that the regenerated bone is of good quality. It is pointed out that the quality and quantity of regenerated bone influence the initial stability of implant and can determine the success of dental implant osseointegration. ${ }^{33-34}$ The new bone density of CGF group was significantly higher than control group. This showed that the osteogenic effect of CGF combined with Bio-oss bone powder in patients with anterior tooth loss is better than using Bio-oss bone powder alone, especially in the early stage. Considering correlation between the bone quality and quantity, future studies about combining platelet concentrates and bone graft materials are needed.

Relaying on the strong soft tissue induction ability of CGF membrane, ${ }^{35}$ a full and sufficient keratinized gingival could be seen 3 months after the operation. The LWHI in the CGF group was higher than the control group 1 week after the operation, indicating that CGF promoted the rapid growth of soft tissue. It was worth mentioning that the VAS score of CGF group on the 1st day after operation was significantly lower than control group, indicating that CGF may relieve postoperative pain. In addition, we found the extraction sites of the two groups healed well after operation, but the attached gingiva in the CGF group were sufficient and plump, while the alveolar ridge in the control group atrophy in varying degrees and the attached gingiva were relatively narrow. The proportion of patients without bone grafting in the CGF group was significantly higher than the control group, suggesting that CGF combined with Bio-oss bone powder can reduce the proportion of patients with bone increment, reduce the cost of bone grafting, shorten the treatment period and create favorable conditions for implant and postoperative aesthetic effect.

\section{Conclusion}

Although long-term studies with large samples are still needed, the following conclusions can be drawn. The use of CGF combined with Bio-oss bone powder in the anterior tooth region can help to reduce postoperative pain at the early stage of healing, form sufficient keratinized gingival tissue and effectively maintain the bone mass of alveolar bone in the three-dimensional direction. Meanwhile, it can provide good conditions for implant repair in the future, and reduce the need for bone grafting before implantation.

\section{Declarations}

\section{Funding}

This research did not receive any specific grant from funding agencies in the public, commercial, or notfor-profit sectors.

\section{Conflicts of interest}


None.

\section{Acknowledgment}

None.

\section{Availability of Data and Materials}

The datasets used and analysed during the current study available from the corresponding author on reasonable request.

\section{References}

1.Kim JJ, Ben Amara H, Chung I, et al. Compromised extraction sockets: a new classification and prevalence involving both soft and hard tissue loss. J Periodontal Implant Sci. 2021 Apr;51(2):100-113.

2.Al Yafi F, Alchawaf B, Nelson K. What is the Optimum for Alveolar Ridge Preservation? Dent Clin North Am. 2019 Jul;63(3):399-418.

3.Stumbras A, Kuliesius P, Januzis G, et al. Alveolar Ridge Preservation after Tooth Extraction Using Different Bone Graft Materials and Autologous Platelet Concentrates: a Systematic Review. J Oral Maxillofac Res. 2019 Mar 31;10(1):e2.

4.Canellas JVDS, Ritto FG, Figueredo CMDS, et al. Histomorphometric evaluation of different grafting materials used for alveolar ridge preservation: a systematic review and network meta-analysis. Int J Oral Maxillofac Surg. 2020 Jun;49(6):797-810.

5.Corning PJ, Mealey BL. Ridge preservation following tooth extraction using mineralized freeze-dried bone allograft compared to mineralized solvent-dehydrated bone allograft: A randomized controlled clinical trial. J Periodontol. 2019 Feb;90(2):126-133.

6.Kamadjaja DB, Abidin ZZ, et al. In Vivo Analyses of Osteogenic Activity and Bone Regeneration Capacity of Demineralized Freeze-Dried Bovine Bone Xenograft: A Potential Candidate for Alveolar Bone Fillers. Int J Dent. 2021 Aug 3;2021:1724374.

7.Shim JY, Lee Y, Lim JH, et al. Comparative evaluation of recombinant human bone morphogenetic protein-2/hydroxyapatite and bovine bone for new bone formation in alveolar ridge preservation. Implant Dent 2018;27:623-9.

8.Rupawala TA, Patel SM, et al. Efficacy of Sticky Bone as a Novel Autologous Graft for Mandibular Third Molar Extraction Socket Healing - An Evaluative Study. Ann Maxillofac Surg. 2020 Jul-Dec;10(2):335343. 
9.Fan Q, Zeng H, et al. Ridge preservation of a novel extraction socket applying Bio-Oss ${ }^{\circledR}$ collagen: An experimental study in dogs. J Dent Sci. 2021 Jul;16(3):831-839.

10.Nagata K, Fuchigami K, Kitami R, et al. Comparison of the performances of low-crystalline carbonate apatite and Bio-Oss in sinus augmentation using three-dimensional image analysis. Int $\mathrm{J}$ Implant Dent. 2021 Mar 23;7(1):24.

11.Gürsoy K, Teymur H, et al. Effect of Platelet-Derived Concentrated Growth Factor on Single-Layer, MultiLayer, and Crushed Onlay Cartilage Grafts. Aesthet Surg J. 2021 Apr 12;41(5):537-547.

12.Takeda Y, Katsutoshi K, et al. The Effect of Concentrated Growth Factor on Rat Bone Marrow Cells In Vitro and on Calvarial Bone Healing In Vivo. Int J Oral Maxillofac Implants. 2015 Sep-Oct;30(5):1187-96.

13.Kim JM, Sohn DS, Bae MS, et al. Flapless transcrestal sinus augmentation using hydrodynamic piezoelectric internal sinus elevation with autologous concentrated growth factors alone. Implant Dent. 2014 Apr;23(2):168-74.

14.Mirković S, Djurdjević-Mirković T,et al. Application of concentrated growth factors in reconstruction of bone defects after removal of large jaw cysts-the two cases report. Vojnosanit Pregl. 2015 Apr;72(4):36871.

15.Kim TH, Kim SH, Sándor GK, et al. Comparison of platelet-rich plasma (PRP), platelet-rich fibrin (PRF), and concentrated growth factor (CGF) in rabbit-skull defect healing. Arch Oral Biol. 2014 May;59(5):5508.

16.Pippi R. Post-Surgical Clinical Monitoring of Soft Tissue Wound Healing in Periodontal and Implant Surgery. Int J Med Sci. 2017 Jul 18;14(8):721-728.

17.Aribau-Gumà C, Jorba-García $A$, et al. Alveolar ridge preservation: an overview of systematic reviews. Int J Oral Maxillofac Surg. 2021 Jul 13:S0901-5027(21)00210-1.

18.Canellas JVDS, Ritto FG, et al. Histomorphometric evaluation of different grafting materials used for alveolar ridge preservation: a systematic review and network meta-analysis. Int J Oral Maxillofac Surg. 2020 Jun;49(6):797-810.

19. Valtanen RS, Yang YP, et al. Synthetic and Bone tissue engineering graft substitutes: What is the future? Injury. 2021 Jun;52 Suppl 2:S72-S77.

20. Bernardi S, Macchiarelli G, Bianchi S. Autologous Materials in Regenerative Dentistry: Harvested Bone, Platelet Concentrates and Dentin Derivates. Molecules. 2020 Nov 15;25(22):5330.

21. Yuan B, Wang Z, Zhao Y, et al. In Vitro and In Vivo Study of a Novel Nanoscale Demineralized Bone Matrix Coated PCL/ $\beta$-TCP Scaffold for Bone Regeneration. Macromol Biosci. 2021 Mar;21(3):e2000336. 
22.Zhao H, Hu J, Zhao L. Histological analysis of socket preservation using DBBM. A systematic review and meta-analysis. J Stomatol Oral Maxillofac Surg. 2020 Dec;121(6):729-735.

23.Sapata VM, Llanos AH, Cesar Neto JB, et al. Deproteinized bovine bone mineral is non-inferior to deproteinized bovine bone mineral with $10 \%$ collagen in maintaining the soft tissue contour postextraction: A randomized trial. Clin Oral Implants Res. 2020 Mar;31(3):294-301.

24.Wang M, Li BW, Wang SW, et al. Preparation and osteogenic effect study of small intestinal submucosa sponge. Beijing Da Xue Xue Bao Yi Xue Ban. 2020 Oct 18;52(5):952-958. Chinese.

25.Lindhe J, Cecchinato $D$, Donati $M$, et al. Ridge preservation with the use of deproteinized bovine bone mineral. Clin Oral Implants Res. 2014 Jul;25(7):786-90.

26.Fiorillo L, Cervino G, Galindo-Moreno P,et al. Growth Factors in Oral Tissue Engineering: New Perspectives and Current Therapeutic Options. Biomed Res Int. 2021 Jan 6;2021:8840598.

27. Rochira A, Siculella L, Damiano F, et al. Concentrated Growth Factors (CGF) Induce Osteogenic Differentiation in Human Bone Marrow Stem Cells. Biology (Basel). 2020 Oct 30;9(11):370.

28.Vaid T, Kumar S, Mehta R,et al. Clinical and radiographic evaluation of demineralized freeze-dried bone allograft with concentrated growth factor versus concentrated growth factor alone in the treatment of intrabony defects. Med Pharm Rep. 2021 Apr;94(2):220-228.

29.Meng HW, Chien EY, Chien HH. Immediate Implant Placement and Provisionalization in the Esthetic Zone: A 6.5-Year Follow-Up and Literature Review. Case Rep Dent. 2021 Sep 15;2021:4290193.

30.Keranmu D, Ainiwaer A, Nuermuhanmode N, Ling W. Application of concentrated growth factor to autotransplantation with inflammation in recipient area. BMC Oral Health. 2021 Oct 30;21(1):556.

31.Wang F, Li Q, Wang Z. A comparative study of the effect of Bio-Oss ${ }^{\circledR}$ in combination with concentrated growth factors or bone marrow-derived mesenchymal stem cells in canine sinus grafting. J Oral Pathol Med. 2017 Aug;46(7):528-536.

32.Durmuşlar MC, Balli U, Dede FÖ, et al. Histological Evaluation of the Effect of Concentrated Growth Factor on Bone Healing. J Craniofac Surg. 2016 Sep;27(6):1494-7.

33. Hindi AR, Bede SY. The effect of osseodensification on implant stability and bone density: A prospective observational study. J Clin Exp Dent. 2020 May 1;12(5):e474-e478.

34.H H, G W, E H. The clinical significance of implant stability quotient (ISQ) measurements: A literature review. J Oral Biol Craniofac Res. 2020 Oct-Dec;10(4):629-638.

35.Mijiritsky E, Assaf HD, Peleg O, et al. Use of PRP, PRF and CGF in Periodontal Regeneration and Facial Rejuvenation-A Narrative Review. Biology (Basel). 2021 Apr 10;10(4):317. 
Figures

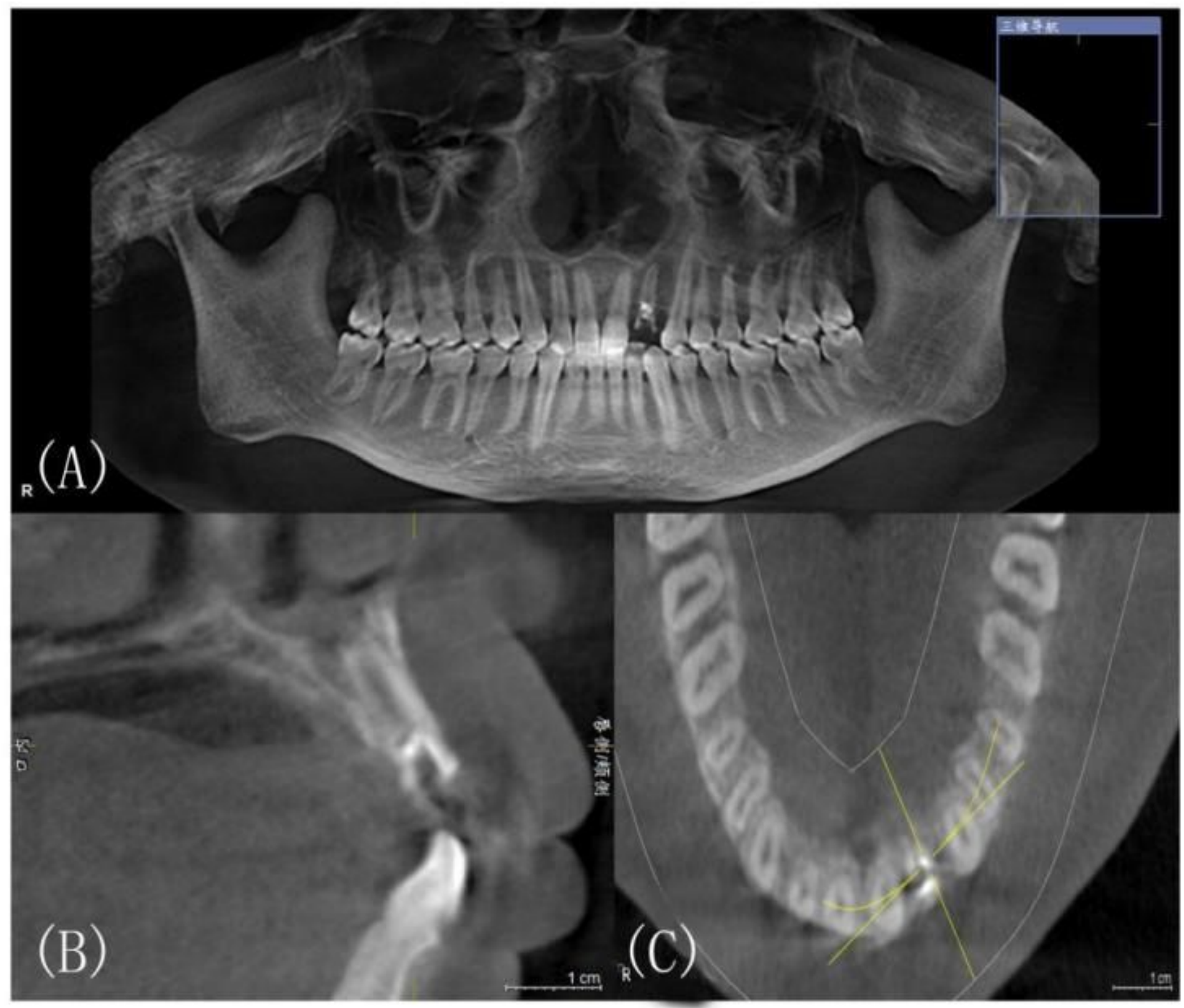

Figure 1

CBCT image of CGF group before tooth extraction 


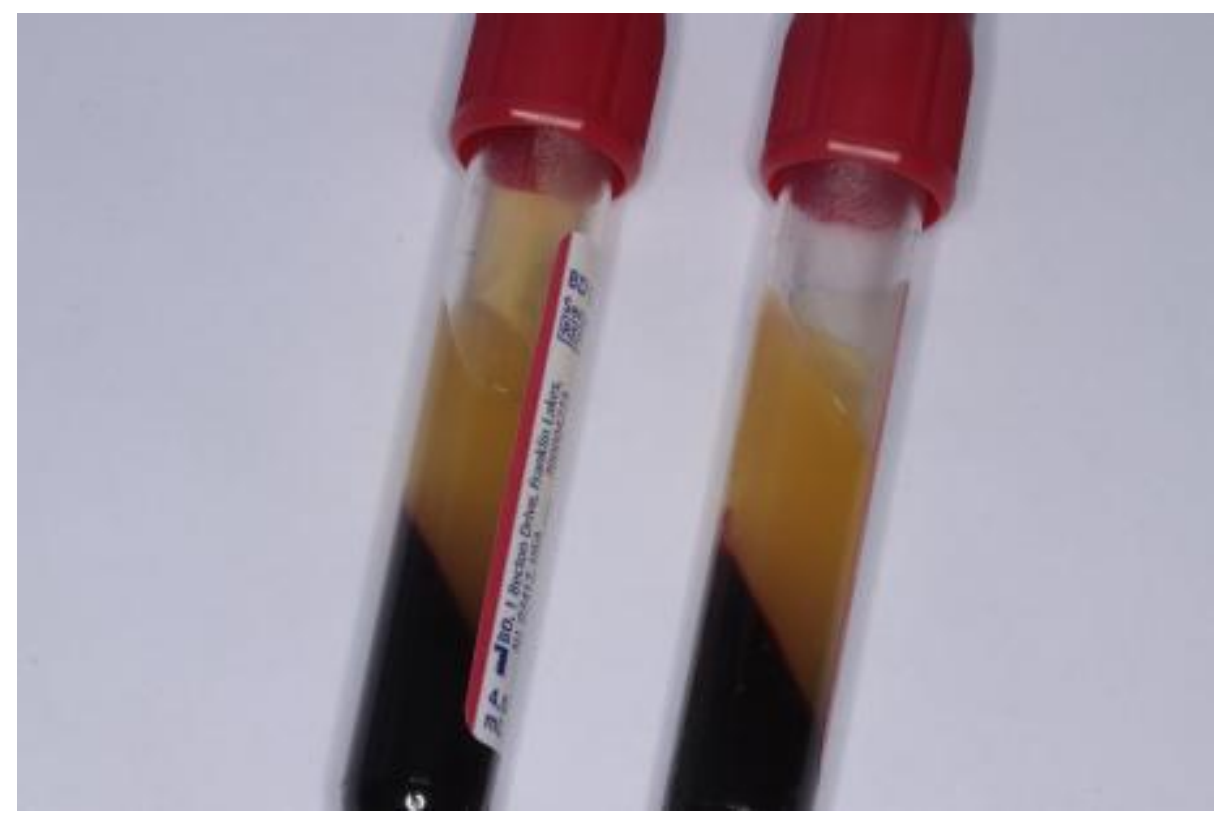

Figure 2

The CGF after separation

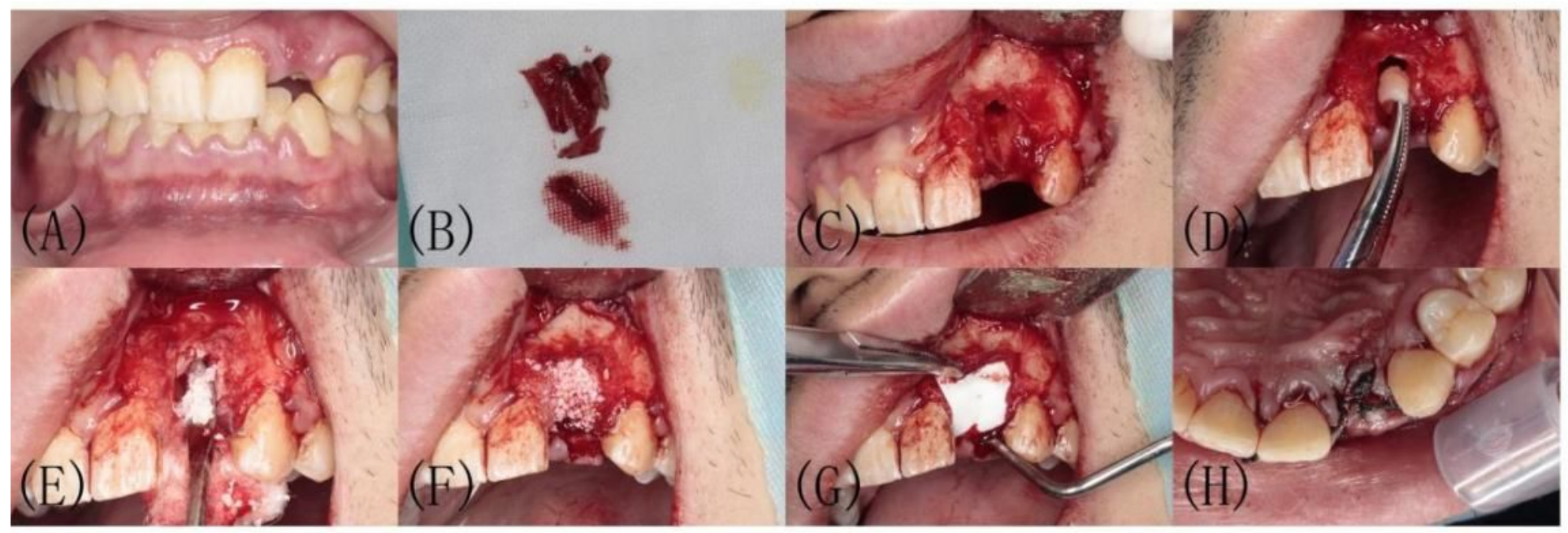

Figure 3

Surgical procedure of CGF group

(A):Labial view before tooth extraction;(B):Extraction and ebridement;(C):Extraction sockets after scoraping;(D)-(E):Small pieces of CGF mixed with Bio-oss;(F): Filled with mixture of CGF and Bio-oss;

$(G)$ :Socket covered with CGF membrane and then collagen membrane; $(\mathrm{H})$ :Contralateral suture of gingival flap. 


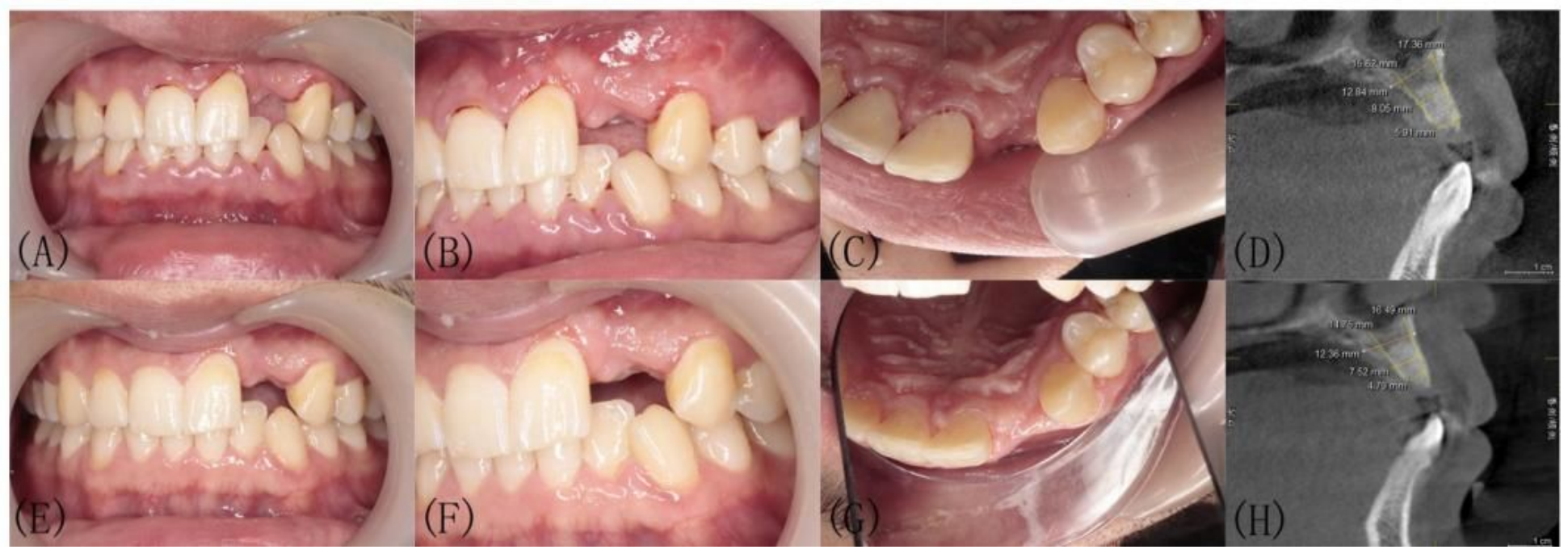

Figure 4

Clinical view and CBCT images of CGF group at 3 and 6 months after operation

(A) -(D): Intraoral and CBCT image of CGF group 3 months after operation; $(E)-(H)$ :Intraoral and CBCT image of CGF group 6 months after operation. 